Portland State University

PDXScholar

$11-15-1972$

\title{
Das Motiv der Einschränkung in einigen Romanen Max Frischs
}

Leen J. Inghels

Portland State University

Follow this and additional works at: https://pdxscholar.library.pdx.edu/open_access_etds

Part of the German Language and Literature Commons Let us know how access to this document benefits you.

\section{Recommended Citation}

Inghels, Leen J., "Das Motiv der Einschränkung in einigen Romanen Max Frischs" (1972). Dissertations and Theses. Paper 952.

https://doi.org/10.15760/etd.952

This Thesis is brought to you for free and open access. It has been accepted for inclusion in Dissertations and Theses by an authorized administrator of PDXScholar. Please contact us if we can make this document more accessible: pdxscholar@pdx.edu. 
AN ABSTRACT OF THE THESIS OF Leen $J$. Inghels for the Master of Arts in German presented November 15, 1972. Title: The Theme of Confinement in Selected Novels of Max Frisch.

APPROVED BY MEMBERS OF THE THESIS COMMITTEE:

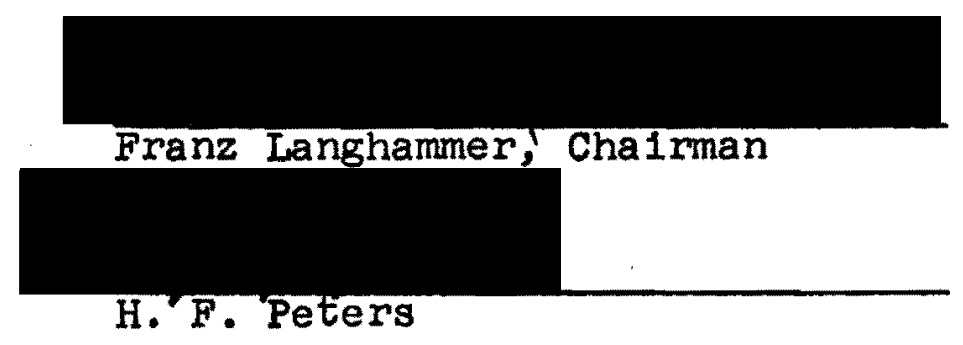

Because of his Swiss origin and background, Max Frisch has personally experienced the confinements imposed upon the Individual by the expectations and demands of society as well as of a national government. He treats the theme of confinement in many of his works, but especlally in his prose.

In agreement with the spirit of his generation, Frisch rejects the claim of soclety upon the individual to conform or to become an integral part of a collective. All human relations which impose upon his way of thinking or acting are rejected and regarded as "claims of imprisonment." Marrlage, class consclousness and government are three forms of imprisonment which serve and enforce the good 
order of soclety, but which also obstruct and almost prevent the possibility for the individual to develop his potential. This point of view as such is not new in the literature of the twentleth century; however, Frisch does not treat the subject of Individualization in an aggressive manner, as do many other contemporary writers. On the contrary, he sees the solution to the problem of confinement in the defensive attitude of the Individual who recognizes and accepts reality, whatever this recognition and acceptance may imply.

Frisch's three heroes try to escape the threefold prison mentioned above: stiller denies his own personality and chooses a new "I"; Homo Faber elevates technique and progress and depends upon the power of cybernetics to gulde his actions; Gantenbein tries to escape reality by playacting different roles or fantasized stories and situations: "Ich stelle mir vor..." (I Imaglne...). All three fail in their attempt to escape and return to their Imprisonment, only to recognize too late that, "Um die Welt zu ertragen, um stand $z u$ halten sich selbst, um am Leben $z u$ bleiben" (In order to suffer the world, to suffer oneself, in order to stay allve) one should face reality in a defensive manner. If reality does not measure up to one's ideals or expectations, the responsibility for change rests upon the shoulders of the Individual, each at his own place and within his abilities. 
In this thesis I examine the reasons for the breakdown in the relations of these three heroes with their environment and compare and contrast their behavior with one another. This is preceded by an attempt to establish the reason why Frisch preoccupies himself so intensely with the theme of confinement and the role which his Swiss background plays in his ideology.

The following three novels were chosen for this study: Stlller, Roman, Fischer Büchere1, Frankfurt a/Main und Hamburg, 1970; Homo Faber, Ein Berlcht, Suhrkamp Verlag, Frankfurt a/Main, 1969; and Mein Name sel Gantenbein, Roman, Fischer Büchere1, Frankfurt a/Main and Hamburg, 1968. Besldes these three novels extensive references are made to Frisch's dlary, Tagebuch 1946-1949, Suhrkamp Verlag, Frankfurt a/Main, 1965. 
DAS MOTIV DER EINSCHRÄNKUNG

IN EINIGEN ROMANEN MAX FRISCHS

$$
\text { von }
$$

Leen J. Inghels

\begin{abstract}
A Thesis Submitted in Partial Fulfillment of the Requirements for the Degree of
\end{abstract}

MASTER OF ARTS

IN

GERMAN

Portland State UnIversity

1972 
TO THE OFFICE OF GRADUATE STUDIES:

The members of the Committee approve the thesis of Leen J. Inghels presented November 15, 1972.

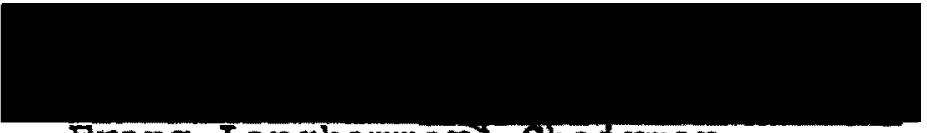

Franz Langhammer, Chairman
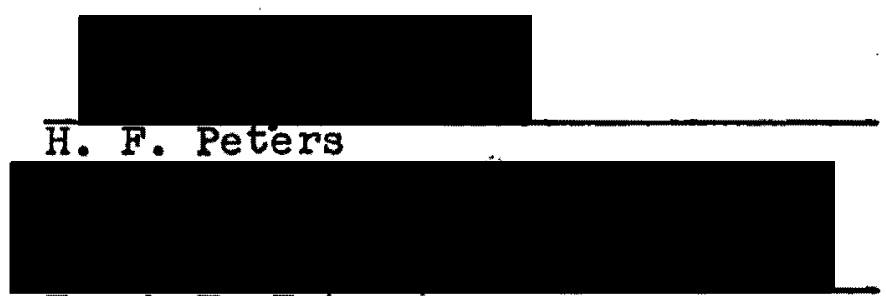

Frank F. Eaton/

APPROVED :

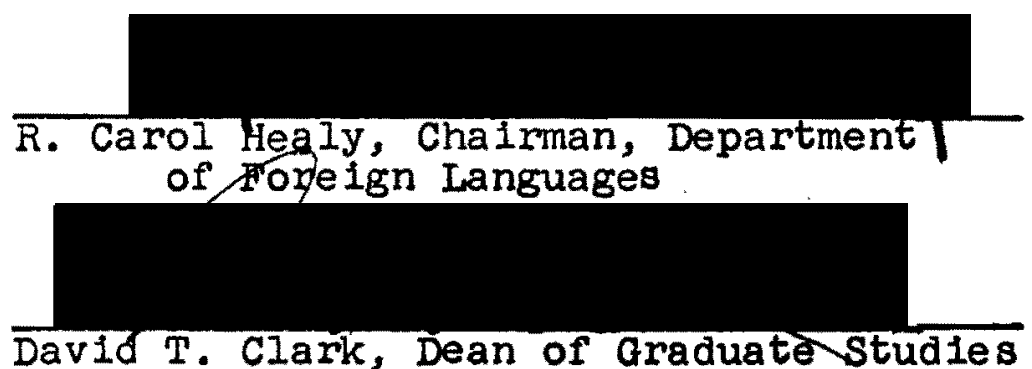

November 15, 1972 
Ich möchte hlermit melne dankbare Anerkennung für den Belstand, der mir bel der Arbelt von Dr. Franz Langhammer und Dr. H. F. Peters geleistet wurde, Ausdruck geben.

Mein besonderer Dank geht aber zu Dr. Walter Dletze und melner Mutter, Frau G. Verrijden, deren Hilfe und Ermutigung bel dieser These unschätzbar waren. 


\section{INHALTSVERZEICHNIS}

SEITE

ANERKENNUNG

KAPITEL

I VERSUCH EINER EINFÜHRUNG IN DIE WELTANSCHAUUNG MAX FRISCHS . . . . . . . . I I

II BESCHREIBUNG DER EINSCHRÄNKUNGEN . . . . . II

III ERKENNTNIS UND SCHEITERN . . . . . . . 24

IV SCHLUSSFOLGERUNG . . . . . . . . . . 39

BENUTZTE IITERATUR . . . . . . . . . . 50 


\section{KAPITEL I}

\section{VERSUCH EINER EINFÜHRUNG \\ IN DIE WELTANSCHAUUNG MAX FRISCHS}

Max Frisch, der infolge seiner schweizerischen Herkunft persönlich viele nationale und gesellschaftliche Elnschränkungen erfahren hat, behandelt das Problem der Einschränkung hauptsächlich in seiner Prosa.

Mit seiner Generation im wesentlichen einig, verwirft Frisch die Forderung nach elner verbindlichen Zugehörigkeit des Elnzelnen zu einem Kollektiv. Alle menschlichen Bezlehungen, die einen Anspruch auf seine Denkens-und Lebenswelse machen, verwirft er und sleht sle als "Ansprüche des Kerkers" an. Ehe, bürgerliche Gesellschaft und Nation sind für thn die drel Kerker, die der Ordnung des Kollektivs dlenen, die aber die Entwicklung des Individuums nahezu völlig verhindern. Diese Ansicht ist an sich nicht neu in der Literatur des 20. Jahrhunderts, Frischs Haltung ihr gegenüber jedoch 1st nicht aggressiv, sondern vielmehr abwehrend, und selne Lösung des Problems der Einschränkung ist eln Sichabfinden mit der Realität.

Immer wieder nimmt Frisch an dem Mangel an Wirklichkeitssinn in den gesellschaftlichen Ordnungen Anstoss. Diese gründen slch Im Geschichtlichen, sind also von der 
Vergangenhe1t bestimmt, und übersehen dadurch das Gegenwärtige, das sich auf die zukunft richten sollte.

Das Wirkliche, meint Frisch, ist die Spannung zwischen Entwurf und Fertigem, "und das Fertige wird stets etwas trostlos sein, unheimisch; alles Fertige hört auf Behausung unsres Geistes zu sein; aber das Werden 1st köstllch". I Zukunft und Entwlcklung sind nur dann möglich, "sobald das Vergangene einmal als vergangen begriffen w1rd." (304) Die Vergangenhe1t 1st also das "Fertıge", und hört auf, "die Behausung unsres Gelstes" zu sein. Das Wirkliche und das Lebendige fehlen den meisten Ordnungen, weil sie sich von der Vergangenhe1t bestimmen lassen. Inre zukunft kann so nur eine Wiederholung sein, und Jede Wlederholung ist, nach Frischs Melnung, tötend.

Entwürfe für die Änderung der gesellschaftlichen Ordnungen sind vorhanden, und die Gegenwart, als Spannung Zwischen Entwurf und Fertigem, gilt für Frisch als das WirkIlche, die "Behausung unsres Geistes".

Dle Verantwortung, um das Kollektiv zur veränderung zu bringen, liegt bel jedem Einzeinen. Die erste Voraussetzung des Werdens wäre wohl der Mut, Nein sagen zu können zu dem, was man als das "Museale", als lebenslose Tradition in seiner elgenen Gesellschaft erkennt.

1. Max Frisch, Tagebuch 1946-1949, Suhrkamp Verlag, 1965, S. 332. Wo diese Elnfuhrung sich mit Max Frischs persönlichen ansichten beschäftigt, werden öfters Hinweise auf sein Tagebuch gemacht werden, und zwar lediglich mit Seitenangaben. 
Frisch 1llustrlert dlese Anslcht mit regelmässigen Hinwelsen auf den Ort seiner Herkunft: die Schwe1z. Als Zugehöriger dleses Staates wird von inm erwartet, dass er seine Nation und ihre Lebensformen bejaht, auch wenn die von 1hr gesteliten Forderungen gegen selne sittlichen Begriffe verstossen. Von selnem Standpunkt aus helsst dies aber "nationalistisch" sein, was er ebenfalls von vornherein abweist; denn für inn 1st eln Natlonalist eln Nih1list, "dem der Mit fehlt, s1ch dazu zu bekennen". (421)

Als Architekt fühlt er sich von "historischer Pletät" bemuttert, "das Neue, das Unsere, 1st Im Grunde schon verworfen, bevor wir unsere Zelchenschrift ergreifen" (192), was er als "Selbstprelsgabe unseres Geschlechtes" benennt. Die Schwelz hat begabte Architekten, die sich aber gezwungen sehen, "dle stadt der Vorfahren" zu erhalten und als. "Reminiszenz" zu pflegen. Sle bauen Geschäftshäuser nach Muster des sechzehnten oder slebzehnten oder achtzehnten Jahrhunderts, verkleiden das Elsenbeton "wie eine Schande mit Quader aus Sandstein, mit St1chbogen und mit echten Erkerlein aus dem Mittelalter".2 Frisch fleht die Architekten an, diese Imitation, diese Mumifikation aufzugeben, wenn sie ihre Helmat noch für etwas Lebendiges halten. Den Künstlern, Schriftstellern und Dichtern geht es nicht andres. Auch in der Literatur 1st das Heimweh nach

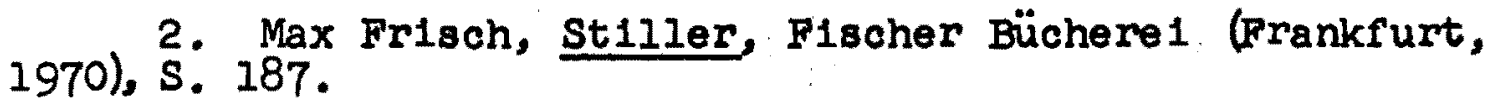


Vorgestern das einzig Annehmbare: "Entführung in die ländIiche Idylle."

Das bäuerliche Leben erscheint als letztes Reduit der Innerlichke1t; die meisten Gedichte melden jede Metaphorik, die der elgenen Erfahrungswelt des Städters entstammen wurde, und wenn nicht mit Pferden gepflügt wird, liefert das Brot innen kelne Poesle mehr; eine gewisse Wehmütigke1t, dass das neunzehnte Jahrhundert immer weiter zurück-. liegt, scheint die wesentlichste Aussage Im schwe1zerlschen Schrifttum zu sein.3

Und wenn Schriftsteller den Mut haben, die W1rklichkelt, die oft negativ ist, in der Geselischaft aufzuzelgen, werden sie von Rezensenten "zwelten Ranges" glelch als Nihilisten abgestempelt. (201) Obwohl Frisch den Rezensenten das Recht zuerkennt, ihre Empfindungen auszudrücken, versagt er den Künstlern, den Schaffenden nicht die verantwortung für die Krit1k.

Kritik der Schaffenden: Die Schaffenden, denke ich, sind besonders befangen, aber inr Urteil hat einen kostbaren Vorzug: wir kennen die Art ihrer besonderen Befangenhe1t, ausgedrückt in inrem Werk, und vor allem hat ihr Urteil immer etwas Geschwisterliches. Es drängt uns niemals in den Sumpf der Selbstgerechtigke1t, was den Rezensenten so leicht gelingt. (343)

In der Schweiz, die das Glück hatte, während des letzten Krleges neutral bleiben zu kônnen, herrscht sehr stark das Gefühl der Selbstgerechtigkelt, ein Anrecht, welches Frisch gleichstark in Zweifel zleht. Die Schweiz, die das Verbrechen ihrer Nachbarn aus inrer "fünfjährigen Gefangenschaft" als Zuschauer erlebte; war nicht in der

3. Frlsch, St1ller, S. 188. 
Lage, slch selbst zu prüfen. Dles gilt sowohl für dle Nation als auch für den Elnzelnen, und Frisch richtet sich nach dem Sprichwort: Gelegenhe1t macht Dlebe. Er untersagt der Schwelz das Recht auf die Behauptung, sle würde vom Faschlsmus oder vom National-Sozlalismus nicht befallen worden seln. Das zelgt er wohl sehr stark in selnem Werk Andorra, wo die guten Andorraner, die die Judenverfolgung Ihrer Nachbarn anklagten, selber zu Judenverfolger wurden, nachdem sle elnen von ihnen als Jude verdächtigt hatten. "Du sollst dir kein Blldnis machen" (31), nicht von dem Elnzelnen, weder von elner Nation, noch von elnem Volk. Frisch fühlt, dass sie Gesellschaft in abstrakten Begriffen lebt, die sle melstens nicht überprüfen kann. Durch dle moderne Massenkommunikation wird das Denken eingeschränkt, was das wirkliche Sehen und Erleben, aber auch die Möglichkelt, elne elgene Melnung zu entwicklen, verhindert. (220) Er lässt St1ller sagen:

Wir leben in elnem Zeitalter der Reproduktion. Das Allermeiste in unserem persönlichen Weltbild haben wir nie mit elgenen Augen erfahren, genauer: wohl mit elgenen Augen, doch nicht an ort und Stelle; wir sind Fermseher, Fernhörer, Fernwisser (. . .) Und mit dem menschlichen Innenleben ist es genau so. Was für ein Ze1talter! 4

Hiermit hat Frisch einige wlchtige Elnschränkungen in der Nation und in der Gesellschaft angeze1gt. Aber dle Lösung, dle Befrelung von dlesen Einschränkungen findet man nicht in

4. Frisch, Stiller, s. 141. 
der Auswanderung. Frisch, der auf seinen Relsen mit vielen anderen Ordnungen in Kontakt kam, deutet auf andersartige Situationen. Er klagt zum Belsplel die amerikanischen Oberflächl1chke1ten an, Ihren obszönen Jugendlichke1tskult, und thre unangebrachte Haltung als "Schutzherren der Welt"5; be1 einem Besuch an eine Ausstellung sowjet1scher Kultur in Berlin klagt Frlsch über das Unbehagen, hervorgerufen von "Jeder Macht, die slch selber prelst" und von dem offensichtlichen Misstrauen dem Westen gegenüber (210-212); in Wlen ärgert er s1ch darüber, dass slch dlese Kultur mit Ihrer "undurchdringlichen Charme" nicht darum kümmert, wie es anderswo aussieht. (234)

Die Lösung wäre vlelmehr ein Sichabfinden mit der Realität. Die Veränderung wird dann elne "elgene Sache", eine Verantwortung, und nicht vom Kollektiv bestimmt. Frisch meint hier slcherlich nicht, dass das Individuum sich absondern, und mit der Flucht in sich selbst (wie zum Beisplel be1 Hesse) die ersehnte Fre the 1t suchen soll, sondern in aller Ehrlichkelt der Gesellschaft entgegentreten und ihr Ihre Unzulänglichkelten entgegenhalten soll. Dass man dazu viel Mut aufbringen muss, bestreitet Frisch nicht, denn Ehrlichke1t in diesem Sinn he1sst "elnsam sein", sagt er. (408) Kelner wird gern mit elner negativen Wahrhelt über slch selbst konfrontlert, ob es sich dabel um eln Kollektiv

5. Max Frisch, Homo Faber, Ein Bericht, Suhrkamp Verlag (Frankfurt a/Main, 1969), S: 220 . 
oder um ein Individuum handelt. Bellebt macht dieser Mut nlcht, und daher die Einsamkelt desjenlgen, der diese Ehrlichke1t zu seiner Elgenschaft macht.

Die Verantwortung des Elnzelnen erhält so elne viel tlefere Bedeutung; nicht nur trägt er sie für selne elgene Entwlcklung, sondern auch: für dle gesellschaftlichen Veränderungen, die für das Lebendigbleiben selner Nation notwendig sind. Und nur so 1st "die Spannung", das elnzig Wirkliche als Bürgschaft für dle Zukunft mögl1ch.

In diesem Sinn gesehen neglert Frisch die Zugehörigkelt des Individuums zu elnem Kollektiv nicht, sondern er gibt dem Individuum die Verantwortung einer Wahl.

Erst aus der möglichen wahl gibt slch die Verantwortung; die Schuld oder die Freihe1t; die menschliche Würde (...). Warum verneinen wir gemeinsam die wirtschaftilche ordnung, die herrschende? Well sle elnem Menschen oder einer Gruppe von Menschen oder der Mehrzahl aller Menschen schlechterdings kelne Wahl lässt; well sle gegen dle wïrde des Menschen verstösst. (165-166)

Der Mensch soll also selner Umgebung abwehrend entgegentreten. Hierin zelgt Frisch seine positive Auffassung. Als Vorbild nimmt er die Italiener, die inn mit Zuversicht auf Ihre Zukunft erfüllen, gerade durch ihre wahl zur Selbstkrltik. "Wleder der unverblümte Mut, das menschliohe Versagen an der elgnen Nation aufzuzeigen." (331)

In der Ehe, als Kelmzelle elner Angehörigke1t, sleht Frisch ebenfalls ein grosses Entw1cklungshindernis. Man sollte hier aber von vornherein andeuten, dass er diese Haft 
nur vom männlichen Standpunkt aus betrachtet und diskutiert. Selne Männer lelden unter dem Druck elnes solchen Bündnisses, der Verantwortung oder des Endgültigen wegen; während die Frauen sich besser in ihrer Rolle als Partner elnzufügen scheinen. Dass stammt wohl aus Frischs Ansicht, dass das Welbliche der Drang 1st, zu sein (daher 1st die Frau auch eine bessere Schausplelerin als eine Dichterin), und das Männliche der Drang, zu tun. (321)

Se in Werk "wimmelt" von Ehemännern, die "weniger die Frau fürchten als die Pflicht, ihr Rechenschaft ablegen zu müssen (. . .), von Junggesellen, die die Verbindlichkelt der Ehe fürchten (...), von Menschen, die das Bildnis, das man von ihnen macht, drückt wle ein Kreuz; wie ein Fluch." 6

Das Bildnismachen steht auch hier wieder als ein erstarrender Einfluss, eine Hinderung zum Wirklichen. Nur In der zeit der grossen, der sogenannten blinden Liebe, nimmt man den Menschen, den man liebt, so, wie er wirklich ist, kann man von Ihm nichts Bestimmtes aussagen, "wir lieben ihn elnfach". Nur zu jener Zelt hält dle Llebe "uns in der Schwebe des Lebendigen, in der Beréltschaft einem Menschen zu folgen, in allen seinen möglichen Entfaltungen". (31) Danach aber dominlert meistens das Bildnismachen wieder. Man schrelbt dem Gellebten Eigenschaften zu, die er

6. Joachim Kalser, "Max Frisch und der Roman. Konsequenzen eines Bildersturms", in Über Max Frisch, ed. Thomas Beckermann, Suhrkamp Verlag (Frankfurt a/MaIn, 1971), S. 4445. 
vielleicht nicht hat, dann ist er dem Blidnis nicht

gewachsen, und das Verhältnis wird öde, zum Stehenbleiben oder sogar zum Zurückgang verurte1lt. Dle Enttäuschung setzt ein, und das Lebendige, das die erste Voraussetzung des Werdens ausmacht, w1rd Im Kelme erstickt.

Oder, sagt Frisch, der Einfluss des Bildnisses kann sich auch im widerspruch zeigen, "dass man so nicht sein w111, wie der andere uns elnschätzt. Man wird das Gegente11, aber man wird es durch den andern." (33) Damit legt er wleder die Verantwortung auf die Schultern des Partners, der durch sein erzeugtes Bildnis ein welteres "glied in jener Kette" 1st, "die inn fesselt und langsam erwürgt". (34) Die elnzige Bedingung, um ein Scheltern der Ehe zu verhindern, ist die überwindung der Vorurte1le. Durch das Vorurte1I versagt man dem Ind1viduum dle grösste Würde eines Menschen: die Wahl, es selbst zu sein. (220)

Wle aus dem Vorhergehenden folgt, zeigt Frisch, dass es "um die Welt zu ertragen, um standzuhalten sich selbst, um am Leben zu bleiben", 7 elgentlich zwel grosse Bedingungen gibt: die Verantwortung vor sich selbst, selner Nation und den Mitmenschen ehrlich zu sein; und die Frelhelt zur Wahl.

7. Max.Frisch, Aus seiner Rede "öffentlichkelt als Partner," in öffentlichke1t als Partner; Suhrkamp Verlag. (Frankfurt, 1967), S, 57. 
Damit sind dle dre1 wichtigsten Einschränkungen, die Frisch als Hindernis für die Entwlcklung der Individuen sleht, angedeutet.

Aus diesem dreifachen Kerker versuchen selne Helden auszubrechen: St1ller durch die Verleugning selner Ident1tät und die Wahl elnes neuen Ichs; Homo Faber durch die Erhebung der Technik; Gantenbe in durch das Rollensplelen und die von seiner Fantasie erzeugten Geschichten und Situationen. Aber alle dre1 scheltern in inrer Flucht und kommen in Ihren Kerker zurück, um zu erkennen, alle zu spät, dass sle sich mit der Realität hätten abfinden sollen.

Es 1st dle Aufgabe der nächsten Kap1tel, das Warum des Scheitern der Bezlehungen dieser dre1 Hauptpersonen zu 1hrer Umgebung zu untersuchen und ihre Haltungen miteinander zu verglelchen und elnander entgegenzusetzen. 
KAPITEL II

BESCHREIBUNG DER EINSCHRÄNKUNGEN

In Stiller und Faber hat Frisch zwe 1 ganz verschiedene Charaktere geschildert. Belde sind typische Produkte des 20. Jahrhunderts: der Aussenseiter (outsider) und der nüchterne Ingenieur. Beide haben sich der Realität nicht anpassen können, obwohl dies auf ganz verschledene Art und Weise geschieht.

St1ller lst der empfindsame Künstler, der Bildhauer, der sich in Gips und Lehm $z u$ verwirklichen sucht, in der Kunst bleibt er jedoch wenig erfolgreich; er verdient nicht genug Geld für seinen Lebensunterhalt; selne kränkliche Frau Julika begreift inn nicht und kann sich für seine Kunst "wo er doch jahrelang ungefähr am Glelchen arbeitete", I nicht begeistern; Stiller ist begabt, aber was er darstellt, unterscheldet slch nicht von dem, was in andern Bildhauerateliers in München, Paris, oder New-York geschaffen wurde. Er ist davon überzeugt, dass nlemand sein Genle auch nur ahnt, und "war fleissig wie ein gepeitschtes Tier, von Ehrgeiz gepeltscht", 2 beglerig auf die Anerkennung seiner

1. Frisch, Stiller, S. 82.

2. Ibid., S. 253. 
Umwelt. Dlese Selbstüberforderung zelgte slch auch, als der junge Stiller damals, während der spanischen Revolution, als Frelwliliger unfählg war, auf den Felnd zu schiessen: auf elnmal sah er in den verhassten Faschisten elnfach vier Menschen. Auch dieses Erlebnis ist für ihn eln Zeichen selnes Versagens und wird von den meisten selner Freunde ebenfalls so angesehen.

Sein späterer Selbstmordversuch scheltert ebenfalls. Er dachte, dass es, wenn er slch das Leben nimmt, "einfach Schluss 1st, Licht aus, Schluss der Vorstellung". Nachher sleht er aber ein, dass dies nicht der Tod wäre, auch nicht, wenn er wirklich gestorben wäre.

Es war, fade gesprochen, elne grosse Verblüffung, etwa wie wenn man von elner Mauer springen würde, um sich zu zerschmettern, aber der Boden kommt nicht, er kommt nie, es bleibt sturz, nichts welter, ein sturz, der auch wieder gar keiner 1st, eln Zustand volikommener Ohnmacht bel volikommenem wachsein. (...) alles bleibt wie gewesen, nichts vergeht, alles bleibt nun ein für allemal.3

Dass Frethe1t durch Selbstmord errelcht werden könnte, ist eine Illusion, sagt Stiller in der Untersuchungshaft, "einfach ein Sprung in die Nichtigkeit, in ein nle gelebtes Leben". 4 Es wäre höchstens eln Abschwimmen ohne Gesch1chte 5

3. Frisch, Stiller, S. 285.

4. Ibid., S. 66 .

5. Max Frisch, Me in Name sel Gantenbein, Roman, Fischer Bücherel (Frankfurt a/Main und Hamburg, 1968), s. 311. 
Er sleht sich also als Versager in allem und sehnt sich danach, anders zu sein als er 1st.

Mit Faber verhält es sich ganz anders. Er steht mit belden Füssen in der Welt, sehr selbstbewusst; er glaubt nlcht an Fügung oder Schlcksal; als Ingenleur lebt er in elner Welt von Maschinen und Mathematik, in elnem Glauben an die Kybernet1k; alles kann wle geplant und geregelt verlaufen, und die Statistiken sind da, um dies zu beweisen.

Nur technische Entwicklung und Fortschritt sind sein zlel. Be1 der UNESCO in der technischen H1lfe für unterentwlckelte Völker tätig, kommt er auf seinen Dienstreisen mit vielen anderen und sogenannten primitiven Kulturen in Berührung, wofür er sich aber nicht interessiert. Es scheint nur wichtig zu sein, ob Strom vorhanden 1st, damit man sich rasieren kann; und wo die Mayas, zum Belsplel, keine Technik haben, sind sie dem Untergang geweint. 6 Er macht sich nichts aus Kunst: weder aus Romanen, noch aus Geschlchte, noch aus Folklore.

Vom Anfang des Buches an wird er durch den Gebrauch genauer Angaben charakterisiert: er reist nur mit einer "Super-Constellation", dle um so-und soviel Uhr, an dem und dem Tag, von dem und dem Ort abfliegt, Richtung so und so, In der und der Höhe fliegt. "(...) wir leben technisch,

6. Erisch, Homo Faber, S. 53. 
der Mensch als Beherrscher der Natur, der Mensch als Ingenleur (. . .) was helsst Schicksal."7

In dem Buch Me1n Name se1 Gantenbe in 1st die Persönlichkelt des erzählenden Ichs nicht so lelcht zu ermitteln. Frisch verhelmlicht diese hinter drel anderen Charakteren: Gantenbe in, Enderlin und Svoboda, denen er verschledene Rollen zukennt: dle er In elne Relhe ähnl1cher Erfahrungen versetzt, um objektiv ihre Haltungen und Reaktionen zu analysleren.

Es schelnt sich hier also wohl mehr um das wirkliche Erleben zu handeln, als um eine Person, die sich zu ldentiflzieren versucht; mehr eln "wle hätte Ich mich benommen, wenn 1ch eln anderer gewesen wäre", und das erzählende Ich entwirft in seiner Fantasie Geschichten und Personen (vor allem männliche, die Frau ist Immer Lila, die weggelaufene Frau des erzählenden Ichs), dle es "anproblert wle Klelder", dle aber Immer, man welss es Im Voraus, "dleselben Falten am glelchen Ort" zelgen werden. 8

Gantenbein scheint dem Leben in gewisser Welse objektlver gegenüberstehen zu können: als Blinder richtet er nlcht, er macht sich kelne Vorurtelle von dem, was er gerade sleht, und zwlngt also selne Umgebung nicht sosehr, selber elne Rolle zu splelen. Er welss, dass die Menschen denken,

7. Frlsch, Homo Faber, S. 131.

8. Fr1sch, Mein Name se1 Gantenbe1n, S. 19. 
er könne 1hr Handeln nlcht beurtellen. Daher bewegen sie sich in seiner Gegenwart verhältnismässig frel und ungezwungen. Der grosse Vortell selner Blindenrolle lst die Möglichke1t einer Scheinharmonie mit seiner Umgebung. Die elnzige Gefahr des Blindselns 1st die Verinnerlichung, die Ihm das, was er tut und erlebt, durch andere Sinne empfinden lassen würde. Aber es ist fa nur eine Rolle, die er splelt, und selne Pfelfe schmeckt daher genau so wie früher, nicht "bitter, betäubend wle elne Tablette oder elne EInspritzung". 9

Selne Rolle, die er auf Hochdeutsch antritt, well dies. Ihm eln wlrklicheres Gefühl des Rollensplelens glbt, hat nur Vortelle, denkt er; sle erlaubt $1 \mathrm{hm}$ ebenfalls elne gewisse "Fretheit", er weiss... er "sleht". Auch das, was die andern verschweigen, ihre verwirrungen und nicht ausgesprochenen Gedanken, die in dem Wechseln von Blicken Ausdruck erhalten, kann er sehen.

Das Motiv des Schweigens und des Sehens, so wle der Sprache, splelt überhaupt elne grosse Rolle in den Werken Max Frischs, was Im Laufe dieser These welter untersucht werden soll.

Enderlin ist ein physisch schwacher Intellektueller, der die 1 hm von der Gesellschaft auferlegte Rolle wohl als Hinderung empfindet, inr Jedoch nicht erfolgrelch

9. Frisch, Mein Name se1 Gantenbe in, S. 41. 
entgegentreten kann. Er sucht sich elne elgene Rolle, die dann aber threrse1ts auch wleder gew isse Erwartungen von der Gesellschaft hervorruft.

Selnem Ruf nach Harvard, zum Be1splel, den er slch so ersehnt hat, fühlt er sich nlcht mehr gewachsen und empilndet Ihn jetzt als Schwindel: "ein insgehelmer Anspruch plötzlich so veröffentlicht". Diejenigen, die Enderlin kennen, glauben kaum noch an diesen fuf.

Wer, wie Enderlin, slch elnmal so entworfen hat, dass er sich durch Leistungen legitimieren muss, wirkt Im Grunde nie vertrauenswürdig.10

Den Vortrag für Harvard hat er, aber um der Le1stung in den Augen der Gesellschaft Anerkennung zu geben, muss er die Rolle elnes Gastdozenten splelen können; "was überzeugt, sind nicht die Lelstungen, sondern die Rolle, die elner spleit". 11

Frisch hält 1hm zur Erläuterung elne andere Geschichte entgegen: der Botschafter elner Grossmacht, der wohl elnsleht, dass er gar nicht die Exzellenz 1st, "für dle inn dle Welt $z$ halten vorglbt", aber der deswegen doch nicht zurücktritt. Er gibt sich der Rolle, die von ihm erwartet w1rd. Er macht Karriere, und "was er fortan von sich selber hält, geht die welt nichts an.' Er splelt also (...) weiterhin Botschafter". 12 Für Enderlin ist es wichtig, was

10. Frisch, Mein Name se1 Gantenbe1n, S. 115.

11. Ibld., S. 115.

12. Ibld., S. 115. 
er von sich selber hält, und er kann die jetzt von ihm erwartete Rolle nicht splelen. In den Augen seiner Umwelt jedoch versagt er.

Was inn noch von dem Botschafter unterscheldet ist, dass dieser die Erkenntnis selnes Rollensplelens nicht preisgibt, "er weiss: jede Selbsterkenntnis, die nicht schweigen kann, macht kleiner und kleiner", 13 und er nimmt dieses Gehelmnis mit ins Grab. Dadurch kommt inm die grosse Anerkennung der Gesellschaft zu. Er hat seine Rolle bis zum Ende meisterlich spielen können.

Enderlin ist sich des Unterschieds zwischen Realität und Rollensplelen also wohl bewusst, was aber bewirkt, dass er sich selbst fremd gegenüber steht. (Mit L1la in der Bar. nennt er sich: der fremde Herr.) Er sucht sich andere Rollen, aber nicht in der Gegenwart, woran er vorbel: lebt, nicht in der Realität, sondern in der Vergangenheit, in früheren Vorgängen, oder in der Zukunft, in seiner Hof fnung.

Dass die Zeit für inn nur als Vergangenheit oder Zukunft existlert, zeigt die Art, wie er seine Begegnung mit Lila erlebt: in der Bar versucht er die gegenwärtige Fremaheit in eine zukünftige Vertrautheit $\mathrm{zu}$ übersplelen, am nächsten Morgen erlebt er den vergangenen Abend und die Nacht.14

Svoboda erinnert gewissermasse an Faber: er 1st selbstbewusst, sportlich, und schelnt, wie dieser, mit

13. Frisch, Mein Name sei Gantenbein, S. 116.

14. Wolf Marchand, "Max Frisch, 'Me in Name se1 Gantenbe in'i, in Thomas Beckermann, über Max Frisch, S. 218. 
belden Füssen in der Welt zu stehen. So wle Faber slch Immer sicherer fühlt, wenn er sich rasiert hat, so fühlt Svoboda slch besser, "wenn er den obersten Kragenknopf aufgemacht und die Krawatte etwas gelockert hat." 15 Aber L1la findet thn öde, ausgesprochen öde. 16 Damlt w1Il Frisch wohl sagen, dass or selner Rolle als Ehemann zu sicher ist. Es ist eine the "family-style", mit den dazugehörigen zärtlichkelten, scharfen worten und Entschuld1gungsbitten. Er kann slch gegenüber Lila wle zu elnem Kind verhalten, nicht sarkastisch, fast scherzhaft, aber doch wie zu einer unmündigen Person, was Llla sagen lässt: sogar seine Güte tyrannislert. 17 Das erzählende Ich sieht in inm elnen Menschen, den man am besten mit dem Kosenamen "Bär" bezelchnen könnte.

Gemeint ist wahrscheinlich das Lleb-Patzige, aber, auch das Kräftige und Langsame und Schwere (....) das Tückisch-Drollige elner klelnäugigen Bestle, die plötzlich sehr bösartig und gewalttätig sein kann, unberechenbar. 18

(Besonders in seiner blinden Elfersucht.) Er lässt die Ehe langsam in seinen Händen zerfallen. Auch er, wie das erzählende Ich, wle Enderlin und Gantenbein, kann Illa nicht behalten, und genau darum scheint es sich doch zu handeln.

15. Frisch, Mein Name se1 Gantenbe1n, S. 218. 16. Ibid., s. 162 .

17. Ib1d., S. 218. 18. Ib1d., S. 224. 
Auch in ihrem Verhältnis zu den Frauen unterschelden slch Stiller und Faber sehr. Stiller benimmt slch den Mädchen gegenüber "wle eln Mönch", unerfahren; wleder drängt slch das Gefühl auf, nicht zu genügen:

und wenn dle Partnerin elnmal selne Grenze erspürt hat, verllert er jeden Mut; er 1st nicht bere1t, nicht Imstande, gellebt zu werden als der Mensch, der er 1st, und daher vernachlässigt er unw1llkürlich jede Frau, dle Ihn wahrhaft liebt, denn nähme er Ihre Liebe wirklich ernst, so wäre er fa genötigt, Infolgedessen sich selbst anzunehmen-davon ist er weit entfernt.19

Was Ihn an Julika, seiner krankhaften Frau, anzleht, ist gerade ihre graziöse und fraglle Schönhelt und dle Möglichkeit, dass sie selne Begrenzung erkennen würde. Ihr objektives Nlchtverstehen selner Kunst allerdings könnte auch darauf verweisen, dass sie nur wenig Ansprüche an thn $z u$ stellen gedenkt.

Sle selbst geht ganz in ihrem Ballett-Tanzen auf, das sle abends erschöpft und $z u$ müde für St1ller oder dle Haushaltarbelten zurückkehren lässt. Stiller kann sich in selner Selbstbemitleldung splegeln, aber sich so auch durch Selbstbeschuldigungen tiefer in sein Versagen elnleben. Wäre er kein Versager, so brauchte dle arme Julika nicht Tag für Tag in ihrer Arbelt gesundhe1tlich zugrunde gehen. In Ihrer the herrscht Schwelgen; schon ehe ein Gespräch beglnnt, kann man das Ergebnis erraten. Und wenn 19. Frisch, stiller, S. 192. 
Stilier sich dann doch einmal vergisst, und sle anschreit, so erschrickt Julika nicht so sehr über das, was er sagt, sondern vielmehr darüber, dass er zu vergessen scheint, wer sie eigentilch ist: eine Tochter aus kultiviertem Haus, wogegen er doch nur aus kleinbürgerllchem M1lleu kam, eigentilch aus gar keinem Milieu. 20

In Ihrer Ichbezogenheit ist Julika sich nicht bewusst, wie sie Stiller langsam erwürgt. Er dagegen lst slch sehr klar darüber, dass er an seine Frau wenigstens elnen Anspruch stellt, den sle nioht erfüllen kann: dass sle thn liebe, sle, die schelnbar nur sich selbst $z u$ lieben vermag. Wie ein "Meertierchen, das nur unter Wasser zu selnem Farbwunder gelangt", erhält Julika Ihre Schönhelt nur im Tanz. Tanz ist Ihr Leben! Das Ballett blelbt dle elnzige Môglichkeit ihrer Wohllust. 21 Von ihren vielen Verehrerm, die nach der Vorstellung auf sle warten, fühlt sle slch nur slcher mit Stiller: sle welss, dass er sle in kelner Welse vergewaltigen wird, denn "dazu fehlt 1hm 1rgend etwas".22

Frisch zelgt hier das Bündnis zweler Menschen, die auf "unselige Weise zueinander" passen.

Sle brauchen einander von ihrer Angst her. ob zu Recht oder Unrecht, jedenfalis hatte die schöne Julika eine helmliche Angst, kelne Frau zu sein.

20. Frisch, Stiller, S. 72 .

21. Ib1d., s. 77 .

22. Ib1d., s. 68 . 
Und auch Stiller, scheint es, stand damals unter elner steten Angst, in Irgendeinem Sinn nicht zu genügen.23

Diese Ehe steht unter dem doppelten Motiv der Impotenz und Frigidität, dle belde tötenden Komponenten slnd. Ohne das Lebendige, das alles Werden erst möglich macht, muss dlese Ehe scheltern.

Faber ist nicht verheiratet, aber nur weil ein solches Bündnis nicht in seinen Lebensbild passt. Es werden verschledene Llebesaffairen erörtert, aber Faber flndet sle absurd, sie ekeln thn an. Seine amerikanische Gellebte heisst Ivy, "Efeu . . so helssen für mich elgentlich alle Frauen".24 Nur mit Hanna, elner deutschen Halbjüdin, die 1936 in Zürich Kunstgesch1chte studierte, "war es n1cht absurd gewesen". Er war sogar bere1t, "Hanna zu heiraten, Ich fühlte mich verpflichtet gerade in Anbetracht der ze1t".25 Denn als schwelzerische Staatsangehörige hätte sie der Gefahr, nach Deutschland ausgeliefert zu werden, entgehen können. Ihr Verhältnis wird noch durch die Tatsache kompliziert, dass Hanna ein Kind erwartet, was überhaupt nicht mit Fabers Plänen überelnstimmt. Sle erkennt seine nüchternen, exakten und sogar fast technischen Überlegungen, die 1 hn zu dieser the rührten. Hanna verhindert die Hochze1t Im letzten Augenblick. Über das Kind

23. Frisch, Stiller, S. 69.

24. Frisch, Homo Faber, s. 111.

25. Ibld., S. 55 . 
wird mehr oder wenlger klar beschlossen, dass es nlcht zur Welt kommen möge.

Faber 1st gegen jede Form der Romantik und Romantisierung, ob es sich jetzt um persönliche Bezlehungen handelt, oder um das Empfinden der Natur. Wenn er üppige Tropengegenden beschreibt, sieht er sle nur mit den Augen eines Technikers, und er 1st nur insofern an diesen Landschaften interesslert, als er daran beobachten kann, wie der Fortschritt slch in thnen verwirklicht, und wie sle der Beherrschung des Menschen unterworfen werden. Sonst ist inm dlese "Fortpflanzere1 überall" zuwider.

Die Rolle der Frau in Mein Name sel Gantenbein, gewissermassen auch in stlller und Homo Faber, hat die Hauptfunktion, das erzählende Ich zu umschreiben. Illa 1st ein Spiegel für Gantenbein, Enderlin und Svoboda. ${ }^{26}$ sle ist und ble1bt die weggelaufene Frau des erzählenden Ichs und erscheint nur in den erfundenen Geschichten in einer jewe1ls erfundenen Rolle, mithin nur als Fiktion. Deshalb scheint es wohl die Absicht Frischs zu sein, in diesem Roman das "Erlebnissmuster" Gantenbelns, Enderlins und Svobodas zu prüfen.

Das elnzige, was mit gewisser sicherke it gesagt werden könnte, wäre, dass Gantenbein m1t L1Ia glückl1ch sein könnte, solange er in seiner Blindenrolle verharre. Als er sich

26. Marchand, S. 222. 
dann aber elnzubilden begann, dass Illa sich wirklich in thn verllebte, selne Rolle also unnötig machte, zelgte selne Fantasie inm auch glelch selne Elfersucht (In Wirklichkelt ändert sich Lila ja nicht). Es wird lihm klar, dass sle sich gegenseitig wieder in erstarrenden Blldern festgelegt hatten. Wenn die erste Ilebe elnmal gewichen 1st, kann elne Versöhnung nur Wiederholung bringen, und nach Frischs Melnung 1st jede Wlederholung tötend. 
KAPITEL III

ERKENNTNIS UND SCHEITERN

Einiges haben Stilier und Faber jedoch mitelnander geme in; belde schreiben 1hre Geschlchte im Rïckblick und Tagebuchst1l, nachdem sle zur Einsicht ihres Fehlverhaltens kamen; belde sind ungefähr glelch alt: $40-50$ Jahre, und durch die niedergeschrlebene Selbstkritik wurde innen das Wirkliche, das Unsagbare kiar. Frisch warnt vor der verwechslung zweler Begriffe: Erleben und Dabeisein. ${ }^{1}$ Denn, sagt er, dle Gegenwart als Schnittpunkt zwischen Erwartung und Erinnerung 1st "als solche kaum erlebbar".2 Erst nachdem Stiller und Faber sich von dem "Dabelsein" haben distanzleren können, waren sle in der Lage, das Erlebnis als Erfahrung aufzufassen und sich selbst ehrlich gegenüberzustehen.

Was wichtig ist, sagt Frisch einmal sehr deutlich: das Unsagbare, das We1sse zwischen den Worten, und Immer reden diese Worte von den Nebensachen, die wir elgentlich nicht melnen. Unser Anllegen, das elgentliche, lässt sich bestenfalls umschreiben, und das heisst ganz wörtlich: man schrelbt darum herum. Man umstelit es. Man gibt Aussagen, die nie unser elgentliches Erlebnis enthalten, das unsagbar bleibt; sle können es nur umgrenzen,

1. Frisch, Tagebuch, S. 420.

2. Ib1d., S. 419. 
möglichst nahe und genau, und das Elgentliche, das Unsagbare, erscheint bestenfalls als Spannung zw1schen diesen Aussagen, 3

Frisch vergleicht die Sprache mit elnem Melssel, den der Blldhauer gebraucht, um das Bekannte, den rohen Ste in, wegzuhauen, und elne Gestalt entstehen zu lassen,

Indem sie die Leere, das Sagbare, vortreibt gegen das Gehelmnis, gegen das Lebendige. Immer besteht die Gefahr, dass man das Geheimnis zerschlägt, und ebenso dle andere Gefahr, dass man vorzeitig aufhört, dass man es elnen Klumpen sein lässt, dass man das Geheimnis nicht stelit, nicht fasst, nicht befrelt von allem was immer noch sagbar wäre, kurzum, dass map nicht vordringt $z u$ seiner letzten oberfläche.4

Stiller hört zu früh auf. Sein Erlebnis bleibt "ein Klumpen", er hat das Geheimnis nicht von allem Sagbaren befre1t. Erst im zweiten Te1l des Buches, Im Nachwort des Staatsanwalts, erfährt man, wie er sich selbst akzeptiert, so wie er 1st: gescheitert als Mann wle als Künstler. 5

Faber dagegen meisselt erbarmungslos weiter, bis das Gehelmnis sich inm stelit.

Stiller, der sich selbst als versager in allem erkennt, entflieht selner the, den Forderungen, die die Gesellschaft an seine Kunst stellt, dem Vorurte1l selner Freunde über sein Benehmen in der spanischen Revolution, aber am wichstigsten wohl sich selbst: dem Versager. Er

3. Frisch, Tagebuch, S. 42.

4. Ibid, s. 42 .

5. Hans Mayer, "Anmerkungen zu St1lier," in Thomas Beckerman, Uber Max Frisch, S. 37 . 
versucht die Katharsis in einem andern Ich zu finden. Er flieht nach Amerika, das Land aller Möglichkelten, das Land der legendären "Fre the 1t" und nimmt dort einen sehr alltäglichen amerikanıschen Namen: James White.

Er relst anscheinend viel: In die Staten und auch nach Mexıko und Havanna; er lernt persönlıch die Armut einer Existenz in New-York kennen (dort fand sein Selbstmordversuch statt). Sonst sagt er in der Untersuchungshaft nicht viel über sein Leben in Amerlka, und wennschon, dann meistens als Beweis dafür, dass er nicht stiller 1st.

Er soll sein Leben niederschreiben, und Frisch gebraucht das Schreiben hier nicht als "Kommunikation mit den Lesern, auch nicht als Kommunikation mit sich selbst, sondern als Kommunikation mit dem Unaussprechlichen" . Mit der Wirklichkelt also, die den Schrelber bedrängt und bewegt. 6

Aus dem Nledergeschrlebenen gestaltet sich dann langsam St1llers Umwelt, in dem Welssen zw1schen den Worten lässt s1ch die Wirkl1chke1t deuten, für dle st1ller kelne Sprache hat 7 und mit der er nicht fertig wurde.

"Ich bin nicht stiller", so fängt er seln Plädoyer an, den verzweifelten Versuch, anders zu sein als er ist und de Welt anders zu sehen als sle 1st. Es hilft aber alles

6. Frisch, Stiller, S. 249.

7. Ibid., S. 65 . 
nichts, es gibt kelne Flucht: die Schwelz blelbt die Schwe1z in 1hrer bürgerl1chen und geograph1schen Beschränkthe1t: sle 1st so tüchtig und "sauber", dass sle sogar das Gitterwerk des Gefängnisses abstaubt; leblos in inrer of fenkundigen Sucht nach materleller Perfektion, in 1hrem Verzlcht auf das Grosse, was schllesslich zur Impotenz führt ${ }^{8}$ (daher das unfrele Schaffen der Schaffenden). Es hilft auch nicht, dass er seine Frau Julika nicht zu erkennen vorglbt, denn selne ohnmächtige Llebe zu der friglden Frau erwacht wleder. Das leblose Bündnls wlederholt slch; Julika blelbt unverändert, aber Stiller 1st sich jetzt selnes Versagens bewusst. Er 1st jedoch noch n1cht Imstande $\operatorname{sich}$ zu ändern, blelbt also unverändert auch in selnem Anspruch an Julika. Selbstmitleld nach wle vor: er kann ja nichts dafür, dass Julika n1cht die Frau 1st, dle er hätte glückl1ch machen können; Selbstüberforderung nach wle vor: er hofft lmmer noch anders $z u$ sein als er ist. . Er sleht jetzt langsam eln, dass das Versagen sich nlcht begraben lässt, und dass er, solange er dles versucht, nicht aus dem versagen herauskommt. 9

Stiller hat auf dem Weg zur Selbsterkenntnis nur den ersten Schritt gemacht, doch droht er jetzt auf dieser stufe

8. Frisch, St1lier, S. 186.

9. Ibld., S. 183 . 
stehenzubleiben und sich "In der Melancholie der Selbsterkenntnis" zu begnügen. 10

Frisch sagt durch den Staatsanwalt in St1ller: die weitaus me1sten Menschen werden durch Selbstüberforderung vernichtet. Unser Bewusstsein hat sich 1m Laufe einiger Jahrhunderte sehr verändert, unser Gefühlsleben sehr viel weniger. Daher elne Diskrepanz zwischen unserem inteliektuelien und. unserem emotionellen Niveau. Die meisten von uns haben (...) Gefühle, dle sle von ihrem intellektuelien Niveau aus nicht wahrhaben wollen. Es gibt zwel Auswege, dle zu nlchts führen: Wir töten unsere primitiven und also unwürdigen Gefühle $a b$, soweit als möglich, auf die Gefahr hin, dass dadurch das Gefühlsleben überhaupt abgetötet w1rd, oder wir geben unseren unwürdigen Gefühlen einfach einen anderen Namen. (. .) Wir etiket
tieren sie nach dem Wunsch unseres Bewusstseins.

St1ller gehört zur zwe1ten Gruppe: er gibt seinen "unwürdigen Gefühlen" dle Etlkette: Versagen - mit der dazugehörigen "Art von schlechtem Gew1ssen". Faber dagegen gehört wohl zur ersten Gruppe, die Gefahr läuft, ihr Gefühlsleben abzutöten. In Ihm ist dle Diskrepanz zwischen intellektuellem und emotionellem Niveau sehr deutlich. Er versucht sich zu verwirklichen in vollem Bewusstsein seiner technischen welt und seiner zeit, des 20. Jahrhunderts. So glaubt Faber sich von einer Schutzmauer umstelit, die inm vor allem Unvorhergesehenen behüten soll. Eln Angriff auf dlese Festung kann nur von aussen kommen, denn das einzige, wozu er sich selber fähig glaubt, ist eln sporadisches

10. Frisch, Stiller, S. 306.

11. Ibid., S. 242 . 
Welchwerden, was aber nur Ermüdungserschelnungen sind, "wie belm Stahl". 12 Aber Gefühle? Nein, denn "Ich halte es mit der Vermunft". 13

Homo Fabers Festung w1rd Jedoch von aussen wle von innen angegriffen. Faber, der sich als Weltbezwinger fühlt, dessen Lebensform und Lebensweg planmässig, nüchtern und automatisch verlaufen soll, der nie krank gewesen 1st und daher die Symptome seines Magenkrebses nicht erkennt, wird durch einen Ohnmachtsanfall und zwe 1 defekten Motoren einer Super-Constellation in eine ganze Kette von Geschehnissen getrieben, die das Unvorhergesehene zur Tatsache machen. Er, der Ingenleur, der selbstbewusste Weltbürger, hat kelne Kontrolle mehr, die Technik und die Kybernetik lassen Ihn im Stich.

Mit einer unwahrscheinlichen Zuversicht in der mathemat1schen Wahrschelnlichkeltslehre glaubte er alles Mögliche umgrenzen und berechnen zu können. Alles wird in hohem Grade Zufall: dass das Mädchen, das $1 \mathrm{hm}$ auf dem Schiff begegnet, 1rgendwie an Hanna erinnert, 1st mögl1ch, denn er denkt in Jenen Tagen wleder öfter an sle; als er später. erfährt, dass Hanna Sabeths Mutter 1st, rechnet er mit der Hilfe der zuverlässigen Mathematik, bis die Rechnung aufgeht, wie er sle haben will: Sabeth kann mur das Kind

12. Frisch, Stiller, S. 113.

13. Ib1d., S. 98 . 
Joachims sein. 14 Mit diesem Rechnen lullt er sein Gewissen und sein Bewusstsein ein und lässt es mit dem jungen Mädchen zu elnem Inzest-Verhältnıs kommen. Erst nachdem er Hanna wledersieht und das Mädchen an einem Schlangenbiss gestorben 1st, erfährt er die entsetzliche Wahrhe1t. Auf elner folgenden Dienstreise (Caracas) fängt er an, seinen Bericht Im Rückblick zu schreiben. Aber zur Selbsterkenntnis kommt er durch diese niedergeschrelbene Selbstkritik in der ersten Station des Berichts noch nicht. Frisch sieht Selbstkritik überhaupt als fragwürdig an:

denn Ihre Wonne besteht darin, dass ich mich scheinbar über meine Mängel erhebe, Indem ich sie ausspreche und Ihnen dadurch das Entsetzliche nehme, das zur Veränderung zwingen würde.15

Faber versucht sich über seine Mängel $z u$ erheben durch die Beschrelbung der Kette von Geschehnisse, um so den Leser und sichselbst von selner Unschuld zu überzeugen. Er errelcht jedoch genau das Gegente1l: Je mehr er sich durch Vorb1lder, Tatsachen und Entschuldigungen $z u$ rechtfertigen glaubt, je mehr seine Schuld sich stellt.

Sein erstes Verfehlen ist wohl, dass er Hanna damals nicht gehelratet hat; sein zweites, dass er s1ch überhaupt In ein Mädchen verllebte, das 30 Jahre jünger lat als er (Hanna nennt es "wldernatürlich") ${ }^{16}$; und seln drittes, dass

14. Frisch, Homo Faber, S. 149.

15. Frisch, Tagebuch, S. 405.

16. Frisch, Homo Faber, S. 242. 
er die Möglichke1t, Sabeth se1 seine Tochter, durch ein "Rechnen" be1se1te schob.

So we1t Fabers erster Schritt zur Selbsterkenntnis, aber von elnem Begnügen in der "Melancholle" ist ke ine Rede. "Erst wenn das Grässliche Inbegriffen 1st, beginnt die mögliche Erlösung, die mehr ist als eine vorellige Harmonie".17 Er hat sich jetzt bewiesen, dass er schuldig ist und gibt sich nicht mit elner voreiligen Harmonie zufrieden.

In Me in Name sel Gantenbe in versucht das erzählende Ich die Wirklichkelt in erfundenen Geschichten darzustelien, we1l Jedes Erlebnis unsagbar bleibt, solange wir hoffen, es ausdrücken zu können mit dem wirklichen Belsplel, das uns betroffen hat. Ausdrücken kann mich nur das Beisplel, das mir so ferne ist wie dem Zuhörer: nämlich das erfundene. Vermitteln kann wesentlich nur das Erdichtete, das Verwandelte, das Umgestaltete, das Gestaltete(. . . $)^{18}$

Um sich seines Erlebnismusters klar zu werden, erfindet sich Jeder Mensch eine Geschichte, die er dann, oft unter gewaltigen Opfern, für sein Leben hält, oder eine Reihe von Geschichten, die slch mit Ortsnamen und Daten durchaus belegen lassen, so dass an ihrer wirklichke1t nicht zu zweifeln 1st.19

Frisch sagt hier wohl sehr deutlich, dass es sich so um eine Erfindung handelt, und nicht um die Wirklichkeit,nicht

17. Frisch, Tagebuch, S. 195.

18. Ibid., s. 411.

19. Max Frisch, Ausgewählte Prosa, Harcourt, Brace \& World, New Yoxk, 1968, S. 10.. 
um eine echte Erfahrung. Obwohl slch dle Geschlchte wohl In früheren Vorgängen zu gründen scheint, blelbt das echte Erlebnis verhüllt. Das elnzig sichere von der vergangenhelt Im Gantenbein-Buch 1st, dass ein Mann in seiner verlassenen Wohnung über selne Erfahrung sinnlert, sle aber nlcht fassen $z u$ können scheint, und sle in "Entwürfe $z u$ elnem Ich"20 umwandelt: "Ich stelle mir vor".

Obwohl dle dre1 Entwürfe des neuen Ichs, Gantenbeln, Enderlin und Svoboda, wle schon gesagt, sehr verschleden vonelnander sind, haben sle jedoch elns gemein: kelner von Ihnen will elne grosse Rolle splelen. Sle verstecken sich hinter ihrer Passivität, hinter ihren Ohnmachtsgefühlen oder ihrer Elfersucht, und scheitern in ihren menschlichen Bezlehungen.

Dle Passivität wird wohl am besten 1llustrlert Im Gantenbein-Charakter. Se ine vermeinte Blindheit glbt ihm alle Möglichkeiten dazu: er lässt sich von seiner Frau aushalten, kann inr höchstens das Bad bere1ten oder in ihrer Abwesendhe1t die Wohnung aufräumen (nicht zu viel, sonst merkt sle es ja). Von thren Freunden erhält er Anerkennung, we1l er so geschickt wein elnschenkt oder eine Forelle zerlègt. Es herrscht eine Scheinharmonie in seiner Ehe, well er vorgibt, nicht zu sehen, dass seine Frau ihn betrügt.

20. Frisch, Mein Name Se1 Gantenbein, S. 117. 
Auch bel Enderlin spielt die Passivität elne Rolle, Jedoch nur, nachdem er erkennt, dass er der aktiven Rolle, die jetzt von thm erwartet wird, nicht gewachsen 1st. Be1 $1 \mathrm{hm}$ ist es die Erkenntnis seiner Ohnmacht, die inn sche1tern lässt. Er kann der Gesellschaft nlcht entgegentreten und wird als Gastgeber Inmitten eines ausgelassenen Fests, das sein Versagen vor dem Durck der Gesellschaft symbollslert, vom erzählenden Ich entlassen. "Ich habe Enderlin aufgegeben". 21

Langeweile und Gewohnheit sind andere Ausdrücke der Passivität, die in der Umschrelbung des griechischen Mythos von Philemon und Baucls charakterislert werden. Dleser Mythos erscheint zur Einleltung in Svobodas Rolle: elne Ehe "family-style". Aber Langewelle und Gewohnheit sind Elnschränkungen, die nlcht das Lebendige innehaben. "Dle Gegenwart 1st dünn, we1l sle abgetragen wird von Tag zu Tag, und die zukunft 1st Altern". 22

Vor dem Altern fürchtet Philemon-Svoboda sich, und er blldet slch eln, dass er Baucis nicht mehr genügt. Zuerst folgt darauf das Misstrauen, dann setzt die Elfersucht ein. Man welss, "dass Eifersucht begründet oder unbegründet, noch selten durch die würde stiller Beherrschung getilgt

21. Frisch, Me1n Name se1 Gantenbe1n, S. 156. 22. Ib1d., s. 133. 
worden ist, eher schon durch eine elgene Untreue".23

Svoboda versucht sich $z u$ verlieben, aber die Frauen wittern seine Eifersucht, "Svoboda hat jetzt den Geruch eines kranken Tlers, und dle Natur 1st gegen 1hn".24 In seiner Ohnmacht kommt er zur Einsicht, was die Zukunft für inn bedeutet, "genauer gesagt: nicht die zukunft, aber das Ende elner Vergangenhelt, die in kelne Gegenwart mehr mündet".25 So wird auch Svobodas Erlebnismuster als möglicher Ehemann für Lila aus der Geschichte entlassen.

Es ble1bt Gantenbein, der jetzt zur Erkenntnis kommt, dass Illa inn nicht mehr betrügt, dass sle thn sogar liebt; dafür hat Gantenbeln aber keine Rolle. ${ }^{26}$ Seine elgene Blindenrolle, wie gesagt, wird dadurch absurd, er will diese sogar aufgeben. Aber die bisher gesplelte Rolle hat die "blinde" Llebe getötet; eln neuer Anfang wäre Wlederholung, und jeder. Wiederholung fehlt das Lebendige: Wlederholung tötet.

Den Prozess, dass Llebe "blind" macht und thr Verslegen "sehend", kann keine Rolle unterlaufen (dabel das typisch Frischsche Paradox, dass man in blinder Liebe mehr sleht als in vermelntilch sehend gewordener, wenn sich elnem nur noch das elne verfestigte Bildnis zeigt). 27

23. Frisch, Mein Name sei Gantenbe in, S. 171.

24. Ibid., S. 231 .

25. Ibid., S. 235.

26. Ibld., s. 303.

27. Marchand, S. 221. 
Ohne lebendige Gegenwart mündet die Vergangenhelt nur in Tod, was Frisch in der Ielche charakterisiert, der es begle1tet von den Behörden (Symbol der passiven Gesellschaft), und obwohl sle "noch elnen sehr entschiedenen Willen: zu éntkommen" hatte, 28 doch nicht gelang, "abzuschwimmen ohne Geschichte" 29 (Symbol der Schein-Freiheit in dem Tod).

Das Buch-Ich, belehrt durch die erfundenen Situationen, findet sich mit der Gegenwart $a b$, "alles ist wie nicht geschehen"; er sitzt unter einem Baum, isst Brot und gerösteten Fisch, prüft, ob der Wein kalt 1st, und fügt sich der Einsamkelt: "Leben gefälit mir".30

In der nledergeschrlebenen Selbstkritik war stiller in der "Melancholle der Selbsterkenntnis" stehengeblieben. Aus dem Nachwort des Staatsanwalts erfährt man, dass er erst kurz vor dem Tod selner Frau von selnem "Sucht, überzeugen zu wollen", befrelt wird.31 Er konnte jetzt aus der Resignation heraustreten, nicht zu sein, "was man so gerne gewesen wäre, und zu werden, was man ist". $32 \mathrm{Er}$ kam sehr spät zu dieser Erkenntnis, zu spät. Belm Anblick der Toten

28. Frisch, Mein Name se1 Gantenbe1n, S. 307.

29. Ibid., S. 311.

30. Ibid., S. 311.

31. Frisch, Stiller, S. 291.

32. Ibid., S. 306. 
kommt er zum ersten Mal zum "tiefen, unbedingten Bewusstsein seiner Versündigung"33: er hatte an der Wirklichkelt vorbe1 gelebt; hatte das Lebendige in erstarrenden Bildern festgelegt und getötet.

Auch er lebt fortan in Einsamke1t, aber ohne Überforderungen an sich selbst, noch an seine Umgebung. "Stiller blieb in Glion und lebte allein".34

In der zwelten station der niedergeschriebenen Selbstkritik melsselt Faber an der Gestaltung seines Verfehlens welter. Er ist jetzt von seiner Dienstreise nach Venezuela zurück in Athen und erwartet dort eine Operation, deren Ergebnis von Frisch keinem Zwelfel überlassen wird: Faber wird sie nicht überleben.

Seine Einsicht in das Sterile der technischen Welt wird in seinem Zorn auf Amerika typisiert. Wo er sich früher dem amerikanischen Lebensform elnfügen konnte, so klagt er jetzt Ihre Oberflächlichkelt an; er nennt dle Amerlkaner Blelchlinge und Vitamin-Fresser, eln Coca-ColaVolk. Er realislert, was Amerika (die Technik) zu bieten hat: "Komfort, dle beste Installation der Welt, ready for use, die Welt als amerikanislertes vakuum". 35

Bezeichnend ist auch wohl; dass er sich jetzt nicht mehr des Genauen und des Konkreten so bewusst 1st: er

33. Frisch, Stiller, S. 327.

34. Ib1d., s. 328.

35. Frisch, Homo Faber, S. 220. 
erinnert sich sogar nicht, in welchem Express er nach Athen reiste, nur noch, dass er Im Speisewagen elnen Steinhäger trank und sich in Verzwelflung die Augen ausstechen wollte.36 Mit dieser Parallele zum Ödipus-Motiv wird auch auf eln Erkennen der Möglichkeit des Schicksals angesplelt. Sein Zorn richtet sich jetzt auf sich selbst, und er entschliesst sich, anders $z u$ leben. Erst nachdem er seinen Dlenst bel der UNESCO gekündigt hat, wird er ruhig. 37

Er 1st slch jetzt völlig Im Klaren über das, was wichtig ist im Ieben, "standhalten dem Licht, der Freude (. . .) Im Wissen, dass Ich erlösche Im Licht über Ginster, Asphalt und Meer, standhalten der Ze1t, bezlehungsweise Ewigkeit im Augenblick", 38 obwohl es für ihn ebenfals zu spät 1st. Er hat sogar nicht mehr die Gelegenhelt seine Innerliche veränderung zu prüfen.

In allen drel Werken Max Frischs, St1ller, Homo Faber und Mein Name sei Gantenbein, kommen die Hauptpersonen zu der Erkenntnis, weshalb ihre Beziehungen $z u$ ihrer Umgebung scheiterten und zu der Schlussfolgerung, dass sie sich mit der Realität hätten abfinden sollen; dass sle ihren Selbstüberforderungen erlegen sind und dass die Ehrlichke1t gegenüber sich selbst und den Mitmenschen die wichtigste

36. Frisch, Homo Faber, S. 239.

37. Ibid., S. 245 .

38. Ib1d., S. 247. 
Bedingung ist, "um die welt zu ertragen, um standzuhalten slch selbst, um am Leben zu ble1ben". 39

39. Frisch, "Öffentlichke1t als Partner," S. 57. 
KAPITEL IV

\section{SCHLUSSFOLGERUNG}

Wie es sich Immer wieder in den Werken Max Frischs he rausstellt, trägt das Individuum die verantwortung für selne Bezlehung zur Realität, sowohl für ihr Scheltern, als auch für inre Veränderung.

Aber das Geschehene kann nicht nachgeholt und nicht verbessert werden. Das Damals ist und blelbt eine Tatsache, auch wenn das Individuum es als Fehlverhalten erkennt. "Die Zeit verwandelt uns nicht. Sle entfaltet uns nur". I Dies wird wohl klar in den zitierten Romanen betont. Stiller verwandelt sich nicht: er 1st und bleibt, wer er Immer war; die finglerten Charaktere im GantenbeinBuch ändern slch auch nicht; sogar Faber bleibt Homo Faber bis zum Ende. Auch wenn er sich nicht mehr an gewisse Einzelheiten erinnert, sein Bericht bleibt mit Daten und Zeltangaben chronologisch präzis. Dle letzte Eintragung und damit das Ende des Berichts he1sst: "08.05 Uhr - Sle kommen". 2 .

Aber die zelt hat sle entfaltet; die zelt 1st das Zaubermittel,

1. Frisch, Tagebuch, S. 22.

2. Frisch, Homo Faber, S. 252. 
das unser Wesen auseinanderzleht und slchtbar macht, indem sle das Leben, das eine Allgegenwart alles Möglichen ist, in ein Nacheinander zerlegt; (. . .) elne Abwicklung, die uns nacheinander zeigt, was eigentlich ein Ineinander ist, ein Zuglelch, das wir allerdings als solches nicht wahrnehmen können, so wenig wie die Farben des Lichtes, wenn sein Strahl nicht gebrochen und zerlegt ist. 3

Fabers und Stillers niedergeschrlebene Selbstkritik wäre so das brechende Prisma, das thr Verhalten in ein Nachelnander zerlegt und inr Fehlverhalten ins Bewusstse in bringt. Deshalb hat Frisch auch wohl für diese beiden Romane den Tagebuchst1l gewählt, der dlese Zerlegung fördert, weil das Denken und Handeln des Augenblicks als "Zugle1ch" empfunden, später jedoch als "Nachelnander" untersucht werden kann. Es muss Jedoch beachtet werden, dass belde auch Im Rïckblick schrelben, wobel Ihr "Zuglelch" schon in der vergangenen Zelt liegt. Bei stiller bringt dies manchmal übertriebene Selbstbeschuldigungen in der Beschreibung einer Lage; bel Faber Versuche der Rechtfertigung, die er mit Aussagen wle "ich gebe zu" oder "1ch bestreite nicht" einle1tet. Das Ergebnis bleibt jedoch dasselbe: das Spektrum inres Fehlverhaltens.

Es 1st wohl anzunehmen, dass Frisch nicht der Ansicht war, bloss diese Charaktere zu schildern, sondern dass er sie vielmehr als Belsplele des Verhaltens des Individuums zu einem Kollektiv, beziehungsweise sein Scheitern Im Kollektiv als Illustration vorzeigen wollte. Es 1st

3. Frisch, Tagebuch, S. 22. 
bestimmt nicht zufällig, dass man beim Lesen seiner Romane oder Theaterstücke immer auf seine Behauptungen im Tagebuch 1946 - 1949 oder in seinen öffentilchen Reden zurückgrelfen kann.

Frischs Hauptsorgen bleiben die Einschränkungen, dle die Individuen verhindern, sich ihrer Umgebung anzupassen und sich in derselben wohl zu fühlen. Dass es in jeder Gesellschaft eine Masse gibt, die sich dieser Einschränkungen nicht bewusst ist, oder besser gesagt, sie nicht $\mathrm{zu}$ sehen vorgibt, hat er im Gantenbein-Charakter gezelgt.

Mangel an Fähigkeiten braucht inn nicht zu bekümmern; was die Welt braucht, sind Leute wle Gantenbein, die nie sagen, was sie sehen, und seine Vorgesetzten werden ihn schätzen; die wirtschaftlichen Folgen solcher Schätzung werden nlcht ausbleiben. Seine Anschauungen $z u$ widerrufen oder auch nur zu ändern, bloss well er Dinge sleht, dle seine Anschauungen widerlegen, wird Gantenbein sich hüten, um nicht aus seiner Rolle zu fallen. Er wird eine politische Karriere machen, nicht eine effektive, aber eine ehrenvolle; er wird überall dabei sein, gestützt auf sein schwarzes Stöcklein, um nicht zu stolpern, und da es elnmal ausgemacht ist, dass Gantenbe in nicht sieht, was gespielt wird vor seinen Augep, wird man überall gern seine Melnung vernehmen. 4

Das sind sle passiven Parasiten, die elnem Kollektiv dienen, indem sie "Wein einschenken", und das Leben leben lassen. Diese fühlen sich sogar "frei", well die Gesellschaft ihren Vortell schützt, "so dass sie mit ihr einverstanden sind".5 Das sind diejenigen, die sich vor der Verantwortung scheuen,

4. Frisch, Mein Name sei Gantenbein, S. 32.

5. Frisch; Tagebuch, S. 203. 
weil das Risiko, das jede Verantwortung innehat, zu gross ist: das hiesse nämlich, die Gesellschaft nicht mehr als Gebrauchsgegenstand betrachten zu können, sondern sle in Ihren Grundfesten zu erschüttern und auf eln $\mathrm{Zlel}$ in der Zukunft aufmerksam zu machen. Das hiesse auch: "ehrlichsein", was ihrerseits wieder "einsam-sein" heisst. Dazu sind die Gantenbeins nicht bereit, und das 1st das Erstikkende, das Frisch in jeder Gesellschaft anklagt. Sie hat keine Entwürfe und lebt in ihrer Vergangenheit, in dem "Gross-Gewordenen", in einer steten Angst vor dem Neuen.

Man erreicht bestenfalls, was man schon gehabt hat, und das ist kein Ansporn, ke in Anrelz zur schöpferischen Bewegung. Das hat etwas Rentnerhaftes (... .) als ginge es um eine Angelegenheit der alten Herren.6

Einer solchen gesellschaftlichen Gegenwart fehlt das Lebendige, das als "Spannung zwischen Entwurf und Fertigem" erscheint. Alle Schaffenden (und Frisch richtet sich öfters an dle Künstler, Schrlftsteller und Archltekten) haben Talente, und sollen schon darin ihren Auftrag sehen: in der Wahrhaftigke1t der Darstellung. eln Individuelles Engagement an die Wahrhaftigkeit, (. . .) ein immer wieder zu leistender Bann gegen die Abstraktion, gegen die Ideologie und ihre tödlichen Fronten, die nicht bekämpft werden können mit dem Todesmut des einzelnen; sie können nur zersetzt werden durch die Arbelt jedes elnzelnen an selnem ort.7

6. Max Frisch, Aus seiner Rede "überfremdung 2 ", in öffentlichkeit als Partner, S. 111.

7. Max Frisch, Aus selner Rede zur Verleihung des "Georg-Büchner-Pre1ses", in öffentlichke1t als Partner, S.55. 
Und das ziel dieses Strebens soll eine Gesellschaft sein, "dle den Geist nicht zum Aussenseiter macht, nicht zum Märtyrer und nicht zum Hofnarren, und nur darum müssen wir Aussenselter unsrer Geselischaft sein, Insofern es keine ist -".8 Also nur insofern dieses $21 e l$ noch nicht errelcht ist, erlaubt Frisch dem Individuum, kein integraler Teil der Geselischaft zu sein, in dem Sinn wie er es für Stiller gebraucht: als Inland-Emigrant, als Mitglied eines Volkes, aber ohne nationale Zugehörigkeltsgefühle. Frisch deutet hier ohne Zweifel auf die lange Reihe von Enlgranten, die von jeher thre Ilebe zum Vaterland bezeugt haben, ehrlich seine Unzulänglichkelten zelgten, und dies mit dem Exil einbüssen mussten. Das Emigrantische wäre dann für Frisch synonym mit dem Lebendigen. So gibt Frisch jedem Einzelnen elgentlich die politische verantwortung der Gesellschaft. Er geht sogar weiter. Im Tagebuch steht:

Wer sich nicht mit Politik befasst, hat die politische Parteinahme, die er sich sparen möchte, bereits vollzogen: er dient der herrschenden Parte1.9

Die grosse Kunst wäre dann, sich politisch $z u$ betätigen, ohne in das Fangnetz der Parte1-Politik zu geraten, in elne Parte1-Überzeugung. Denn ṫberzeugung selber kann wieder zur Einschrănkung werden. In der Überzeugung umgibt man slch wleder mit erstarrenden Bildern, die das Lebendige der
8. Frisch, Tagebuch, S. 62 .
9. Ib1d., s. 329. 
Spannung zwischen Entwurf und Fertigem erwürgen. Was Frisch hler vielmehr meint, ist das Engagement in der Polis: in dem Problem der Lebensgemeinschaft der Menschen, der gesellschaftlichen Ordnungen, "dessen Lösung den Anfang der Kultur darstellte, (. . .) oder den Untergang verursachte" 10 Mit dieser Politik soll der Elnzelne slch beschäftigen: mit den Menschen also, und mit allem Menschlichen, "denn unsere Helmat 1st der Mensch; Ihm vor allem gehört unsere Treue." 11

Die Verantwortung des Elnzelnen geht daher welt über die eigene Gesellschaft hinaus und ist nicht an nationale Grenzen gebunden. Der ort, von dem aus das Engagement für die Menschhe1t gele1stet wird, 1st von keinem Belang, ob der Wohnsitz aus Zufall der Geburt, oder aus freler Wahl entsteht. Ideal wäre sogar, dass der Zufall mit der wahl zusammenhänge, aber wle dem auch 1mmer sei: dieser Wohnsitz soll das unausgesprochene Gefühl der Unzugehörigke it gestatten. 12 So sieht Frisch die Inland-Emigration, und das Emigrantische äussert sich darin,

dass wir nicht im Namen unserer Vaterländer sprechen können noch wollen; es äussert sich darin, dass wir unsere Wohnsitze, ob wir sle wechseln oder nicht, überall in der houtigen welt als provisorisch empfinden.13

10: Frisch, Tagebuch, S. 328.

11. Ib1d., S. 170.

12. Frisch, "Büchner-Rede", S. 51.

13. Ib1d., S. 50 . 
So sleht Frisch die Verantwortung der Individuen zum Kollektiv: akt1v, mit belden Füssen in der Realität, als Emigrant ohne seln Vaterland zu verlassen, verpflichtet an eine Gesellschaft "die den Gelst nicht zum Aussenselter macht". 14 ob das Ziel erreicht wird, oder ob es Utopie bleibt, ist nicht wichtig. Das Engagement wird jedenfalls das Lebendige versichern,

über die Landesgrenzen hlnweg, über die Sprachgrenzen hinweg, über dle Rassengrenzen hinweg verbunden in unsrer Be fahung des elnzelnen, selber nichts andres als einzelne. I

So sleht Frisch die Welt von allen Vorurteilen gesäubert. "Du sollst dir kein Bildnis machen", 16 nicht von dem Einzelnen, nicht von einer Nation, nicht von einem Volk.

Frisch hat in seinen Werken Menschen mit ihren positiven und negativen Qualitäten dargestellt, mit ihren Stärken und mit inren Schwächen; Menschen, die der Realität nicht gewachsen sind, und die slch durch Selbstüberforderung $\mathrm{zu}$ bewerten versuchen oder die an der Realität vorbelleben. Seine Helden sind keine Abstraktionen, sondern Menschen, die selber in Wirklichkelt die Gesellschaft bilden.

Aus seinen Werken kommt ein Ruf zur Verantwortung des Einzelnen, ein Aufruf zur Erkenntnis und Annahme der

14. Frisch, Tagebuch, S. 62.

15. Frlsch, "Büchner-Rede", S. 53.

16. Frisch, Tagebuch, s. 31. 
Wirklichke1t. Das erzählende Ich im Roman Me in Name se1 Gantenbein, sowle Stiller und Faber vermögen nicht die Realität $z u$ erkennen. Im ersten Roman ist sle die verlassene Wohnung, Einsamke1t. In Stiller ist sie die gescheiterte Ehe, oder besser gesagt: die Sterilität und Impotenz. Im Homo Faber 1st es das Relative der Technik. Allen fehlt ein "spontanes Verhältnis zur Realität".17 Sie sehen nicht ein all-einbegriffenes Ganzes und starren blind auf Telle oder Ansätze eines Ganzen.

Der moderne Mensch Faber glaubt, sein Leben nach seinem willen regeln zu können, wenn er nur alles sorgfältig ausrechnete. Das ist sein Tell des Ganzen. Er rechnet nicht m1t den zufälligkeiten des Alltags, die "die Bedeutung von Sch1cksal oder Fügung" annehmen können.

Durch die vordergründliche, rational-technischstatische Auslegung der Weit, die keine Transzendenz mehr kennt, baut Faber sich selbst die Falle, in der er zu Fall kommen muss, wenn etwas von aussen in diese Welt einbricht.18

In den Hintergrund dieses Nicht-Anerkennens des Zufalls hat Frisch das ödipus-Motiv eingebaut, jedoch mit einem sehr wichtigen Unterschled: Ödipus war sich der Macht des Schicksals bewusst und versuchte, thm zu entgehen. "Faber lehnt dle Möglichkelt des Unvorhergesehenen ab, und weigert

17. Frisch, Homo Faber, S. 177.

18. Rolf Geissler, Möglichkelten des modernen Deutschen Romans, Verlag Moritz Diesterweg, Frankfurt a/Main, 1970, S. 195. 
slch, bestimmte Umstände als Zufall anzuerkennen. Wo ödipus nach der Analyse des Geschehens zur Erkenntnis kommt, geht Faber zur Analyse über, nachdem er zur Erkenntnis gekommen 1st; sieine Frage bleibt: wie konnte alles so kommen? Wle ein programmiertes Elektronen-Hirn wägt er die gegebenen Informationen. Das Ergebnis ist: der Mensch ist kein Roboter und hat mit seinen Gefühlen zu rechnen. Das Leben lässt s1ch nlcht durch Kybernetik und Technik allein lenken, aber es soll die Möglichke1t des Zufalls mit elnschliessen.

Für Frisch geht die Gefahr der Erhebung der Technik aber viel weiter. Ein Leben, das die Verantwortung des Einzelnen seinen Mitmenschen gegenüber nicht anerkennt, w1rd zum "amerikanischen Vakuum", die Technik entfernt und verf remdet das Individuum der Realität:

Je ferner, um so lelchter. Das ist der segen unsrer Technik, be1läufig bemerkt; so von Angesicht zu Angesicht, das gebe ich zu, da sind wir alle étwas zimperlich. Wer wäre schon imstande, (....) siebenhundert Menschen elgenhänd1g zu ersäufen, $\left(. .^{\circ}.\right)$ ? Wer schafft das? EIn Torpedo, das 1st doch etwas ganz andres. Ein einziges Torpedo, ein Blick auf die Uhr, ein Druck auf den Knopf; das kann jeder, und wären selnen Augen noch so blau. Was 1st dabe1! Sehen Sie sich elnmal die Jungens an, die die Bomben lösen; ke1n Makel im Gesicht. Was heisst töten? Natürlich an ort und Stelle - aber dazu haben wir ja die Technik, me in Freund, oder wie 1ch zu sagen pflege: man muss grundsätzlich denken, und das gelingt den allermelsten nur dann, wenn sie ihre Tat nicht mit Augen sehen.19

19. Frisch, Tagebuch, S. 361 . 
Frisch zelgt klar, dass die Technik den Einzelnen auch zum Massenmörder machen kann, und entsagt ihm nlcht der Schuld.- Er fleht den Menschen an, die Realität bewusst zu erleben, und sich vom Fernsehen, Fermhören, Fernw1ssen und Fernerleben zu befrelen.

Ausserdem hofft Frisch, dass man nicht nur hört, dass er schreit, sondern auch was er schreit. 20

Man Ilesse Frisch keine Gerechtigke1t widerfahren, wenn man eine Dlskussion über seine Arbeit schliessen würde, ohne etwas über seine Sprache zu sagen. Ob es sich um seine Romane, seine Theaterstücke, sein Tagebuch oder seine Reden handelt, das Iesen wirkt Immer glelch fesselnd. Man braucht nicht mehr Worte, als Hans Mayer: "Die bewegte, klare, genaue und bewegende Prosa Max Frischs zu lesen, ist elne grosse Freude". 21

Max Frisch handelt nach seinen Worten: als Architekt versucht er dle Anslchten seines Vaterlandes zu ändern, es aus dem "Musealen" zu zlehen. Als Schriftsteller weist er das Kollektiv und das Individuum auf Ihre Unzulänglichkelten und inre Verantwortung der Menschhe1t gegenüber hin. Als Politiker wlegt er das Fur und Wider der demokratischen

20. Frisch, Tagebuch, S. 453.

21. Hans Mayer, "Über Max Frisch 'Tagebuch 1966-1971' Die Schuld der Schuldiosen," In Der Splegel, nr. 25, 12. Jun1 1972, S. 124. 
und sozlalistischen Weltanschauugen. Als Individuum ist er slch selner Aufgaben bewusst:

er 1st ein engagierter Mensch! 
Blcknese, Günther. "Zur Rolle Amerikas in Max Frischs Homo Faber", in The German Quarterly, XIII (September 1969), 52-64.

Dürrenmatt, Friedrich. "'Stiller', Roman von Max Frisch, Fragment einer Kritik", in über Max Frisch, ed.

Thomas Beckermann, Suhrkamp Verlag, Frankfurt a/Main, 1971 .

Esslin, Martin. "Max Frisch", in German Men of Letters, Vol. Three, ed. Alex Natan, oswald Wolf, London, i968.

Franzen, Erich. "Über Max Frisch", In Über Max Frisch, ed. Thomas Beckermann, Suhrkamp Verlag, Frankfurt a/Ma in, 1971 .

Frisch, Max. Andorra, Suhrkamp Verlag, Frankfurt a/Main, 1968.

- Ausgewählte Prosa, Suhrkamp ed., Harcourt, Brace \& World, New York, 1968.

- Bledermann und die Brandstifter, Suhrkamp ed., Harcourt, Brace \& World, New York, 1970.

- Bin, oder Die Relse nach Peking, Suhrkamp Verlag,

- Frankin, oder Die Re ise

- Blografle: E1n Splel, Suhrkamp ed., Harcourt, Brace \& World, New York, 1972 .

- Blätter aus dem Brotsack, Atlantis Verlag, Zürich, 1969.

- Homo Faber, Ein Berlcht, Suhrkamp Verlag, Frankfurt a/Main, 1969.

- Meln Name se1 Gantenbein, Roman, Fischer Büchere1, Frankfurt a/Main und Hamburg, 1968.

- Öffentl1chke1t als Partner, Suhrkamp Verlag, Frankfurt a/MaIn, 1967. 
Frisch, Max. Stiller, Roman, Fischer Büchere1, Frankfurt a/Ma in und Hamburg, 1970 .

- Tagebuch 1946-1949, Suhrkamp Verlag, Frankfurt a/Main, 1965.

Gelssler, Rolf. Möglichke1ten des modernen deutschen Romans, Verlag Moritz Dlesterweg, Frankfurt a/MaIn, 1970.

Helssenbüttel, Helmut. "Max Frisch oder Dle Kunst des Schreibens in dieser Ze1t", in Uber Max Frisch, ed. Thomas Beckermann, Suhrkamp Verlag, Frankfurt a/Main, 1971 .

Jens, Walter. "Erzählungen des Anatol Ludwlg Stiller", In Über Max Frisoh, ed. Thomas Beckermann, Suhrkamp Verlag, Frankfurt a/Main, 1971.

Kähler, Hermann. "Max Frıschs 'Gantenbein'-Roman", in Über Max Frisch, ed. Thomas Beckermann, Suhrkamp Verlag, Frankfurt a/Main, 1971.

Kaiser, Joachim. "Max Frisch und der Roman. Konsequenzen elnes B1ldersturms", in Uber Max Frisch, ed. Thomas Beckermann, Suhrkamp Vertag, Frankfurt a/Main, 1971.

Marchand, Wolf R. "Max Frisch, 'Me In Name sel Gantenbein'", in Über Max Frisch, ed. Thomas Beckermann, Suhrkamp Veriag, Frankfurt a/Main, 1971.

Mayer, Hans. "Anmerkungen zu 'Stiller'", In Über Max Prisch, ed. Thomas Beckermann, Suhrkamp Verlag, Trankfurt a/Main, 1971.

- "Über Max Frisch 'Tagebuch. 1966-1971' Die Schuld der. Schuldlosen", in Der Splegel, 12. Jun1 1972.

Re1ch-Ran1ck1, Marcel. Deutsche Literatur in West und ost, Prosa seit 1945, R. Piper \& Co. Verlag, Múnchen, 1963.

Ro1sch, Ursula. "Max Frischs Auffassung vom Elnfluss der Technik auf den Menschen - nachgewlesen am Roman 'Homo Faberi", in über Max Frisch, ed. Thomas Beckermann, Suhrkamp Verlag, Frankfurt a/Ma in, 1971.

Schm1d, Karl. "'Andorra' und die Entscheldung", In über Max Frisch, ed: Thomas Beckermann, Suhrkamp Verlag; Frankfurt a/Ma1n, 1971. 